\title{
Observation of dynamic maximum in a turbulent cascade on the surface of liquid hydrogen
}

\author{
I.A. Remizov, M.Yu. Brazhnikov, and A.A. Levchenko \\ Institute of Solid State Physics RAS, Chernogolovka, Moscow district. 142432, Russia \\ E-mail: levch@issp.ac.ru
}

Received May 17, 2016, published online October 24, 2016

\begin{abstract}
We report on the experimental observation of energy accumulation near the high frequency boundary of the inertial range in the spectrum of turbulence in a system of capillary waves on the surface of liquid hydrogen driven by a harmonic force. The effect is manifested as a local maximum in the spectrum of pair correlation function of the surface elevation. This phenomenon is dynamical and can be seen only during reconfiguration of the turbulent cascade caused by waves generation of below the driving frequency.
\end{abstract}

PACS: 47.27.Gs Isotropic turbulence; homogeneous turbulence.

Keywords: low temperatures, liquid hydrogen, nonlinear waves, turbulence, surface waves.

\section{Introduction}

A wide set of nonlinear wave systems can be described in the frame of the theory of weak wave turbulence [1]. Among them are capillary and gravity waves on the surface of water, Rossby waves in the atmosphere and oceans of planets, Langmuir waves in plasma, and spin waves in magnetics. A relatively low viscosity of liquid hydrogen and the possibility to excite waves on the charged surface of cryogenic liquids by electrical force offer a unique opportunity for experimental studies of wave turbulence. The use of liquid hydrogen for experiments on wave turbulence has already allowed us to study phenomena predicted by the theory, e.g., Kolmogorov-Zakharov steady state spectra of capillary turbulence in a wide range of frequencies, as well to observe new ones which have been explained successfully in the framework of the weak turbulence approximation: quasi-adiabatic decay of capillary turbulence and suppression of high-frequency turbulent oscillations by additional low-frequency driving force [2].

The dispersion of waves on the surface of liquid is given by:

$$
\omega^{2}(k)=g k+(\sigma / \rho) k^{3}
$$

where $\omega$ and $k$ are the frequency and the wave vector of a surface wave, correspondingly, $\sigma$ and $\rho$ are the surface tension and the density of liquid, and $g$ is the free-fall acceleration. The first term in (1) prevails in the long wave region and describes gravity waves, the second one corresponds to capillary waves (ripples).

The main processes of the nonlinear interaction for capillary waves are three-wave processes of decay and merging that satisfy the conservation laws of frequency and wave vector:

$$
\omega_{1}=\omega_{2}+\omega_{3}, \quad \mathbf{k}_{1}=\mathbf{k}_{2}+\mathbf{k}_{3} .
$$

Due to discreteness of the eigenmodes of the surface oscillation of liquid in a finite basin these relations cannot be always satisfied, e.g. for capillary waves in a square basin three-wave processes are prohibited: the set of equations has no mathematical solution [3]. In real systems these resonant conditions are softened due to the viscous and nonlinear broadening of the resonances.

Neglecting viscous and nonlinear effects a radially symmetric surface wave in a cylindrical basin is described by the Bessel function. The boundary conditions on the resonator wall define the eigenvalues of wavenumber $k$ : $J_{1}(k D / 2)=0$, where $D$ is the resonator diameter, $J_{1}(x)$ is the Bessel function of the first order. Using the asymptotic form of the Bessel function for large $k$ we can find that the resonance wavenumbers are approximately equidistant with spacing $\Delta k \sim 2 \pi / D$.

The pair correlation function of the surface deviation from an equilibrium state in the Fourier representation $I_{\omega}$ in the inertial range is described by the power function of frequency $I_{\omega} \sim \omega^{-\beta}$. For the case of broadband high-frequency excitation of the surface with spectrum (1) the value of the index $\beta$ equals 17/6 [1], and narrowband excitation $\beta$ equals 23/6 [4]. 
A nonlinear wave interaction leads to energy redistribution from a low frequency domain, where the energy is injected by an external force, to higher frequencies, where the energy is dissipated due to viscous losses. The position of high-frequency boundary of the inertial range $\omega_{b}$ can be found assuming that a nonlinear energy flux is comparable with the amount of energy leaving the cascade because of viscous damping. In the case of continuous spectrum [5]:

$$
\omega_{b} \sim\left(\eta_{0}^{2} \omega_{0}^{17 / 6} / v\right)^{6 / 5}
$$

where $\eta_{0}$ is the wave amplitude at the pumping frequency $\omega_{0}$. The estimation shows that the high-frequency boundary $\omega_{b}$ for liquid hydrogen at $15 \mathrm{~K}$ and water at normal conditions are related as 5:1 under the same condition of excitation. From this point of view liquid hydrogen is a preferable fluid to study the capillary turbulence.

Turbulent distribution may deviate from the power dependence. One of the possible reasons for the deviation can be associated with a discrete spectrum of surface excitations instead of continuous spectrum (1) [6], which leads to the energy accumulation on several resonance modes near the edge of the inertial interval [7].

The frequency distance between the nearest resonant modes for capillary waves in a cylindrical basin at high frequencies is given by:

$$
\Delta \Omega=(3 \pi / D) \cdot(\sigma / \rho)^{1 / 3} \omega^{1 / 3} .
$$

Strictly speaking, in the ideal system of capillary waves the laws of energy and momentum conservation cannot be satisfied simultaneously. This restriction is removed taking into account the broadening of the resonance peaks due to viscous losses and non-linear interaction of waves. At high frequencies the broadening due to viscosity becomes comparable with the distance between the resonance modes $\Delta \Omega$ at the frequency

$$
\omega_{C} \sim(3 \pi / 4 D)(\sigma / v \rho) .
$$

For example, in our experiments $D=60 \mathrm{~mm}$, the value $\omega_{c} / 2 \pi$ for liquid hydrogen at the temperature of $15 \mathrm{~K}$, and for water under normal conditions equals $4 \mathrm{kHz}$, and $3 \mathrm{kHz}$, respectively.

Another reason for deviation of the turbulent distribution $I_{\omega}$ from the power function may be connected with an insufficiently effective absorption of energy within the dissipation domain due to viscosity. This leads to an increase of the harmonic amplitudes (accumulation of energy) near the high-frequency edge of the inertial interval to keep an energy flux propagated on turbulent cascade constant [8].

In the experiments presented here we were able to observe the accumulation of energy near the high-frequency edge of the inertial interval in the time-dependent (dynamic) conditions.

\section{Experimental technique}

The experimental method of wave registration on the surface of a cryogenic liquid was described in [9]. The investigations were carried out at the liquid hydrogen temperature $T=15 \mathrm{~K}$. Hydrogen gas was condensed into copper cup of the inner diameter $60 \mathrm{~mm}$ and $4 \mathrm{~mm}$ deep. The cup and a copper plate which was fixed $4 \mathrm{~mm}$ above the cup form a flat capacitor. The liquid was ionized with a source of charges placed on the bottom of the cup. The liquid surface is charged with positive ions extracted from the bulk of liquid. dc voltage of about $1 \mathrm{kV}$ is applied between the capacitor plates.

A low-frequency ac voltage applied in addition to the dc voltage excites waves on the charged liquid surface. Waves are detected by means of a laser beam reflected from the liquid surface and then focused into a photodetector. Variation of the angle between the laser beam and the oscillating surface leads to the modulation of the reflected light power. The ac signal from the photodetector is amplified and digitized by a 24-bit analogto-digital converter with a sampling frequency of $102.4 \mathrm{kHz}$. The digitized signal $P(t)$ is proportional to the variation of power of the reflected laser beam. As we showed earlier [9] the power spectrum $P_{\omega}^{2}$ is proportional to the spectrum of the pair correlation function of the surface elevation $\left\langle\eta_{\omega}^{2}>\right.$.

\section{Results}

Oscillations on the surface of liquid hydrogen were excited at the frequency of the fifteenth resonance in the experimental cylindrical cell, $\omega_{0}=58.6 \mathrm{~Hz}$. Figure 1 exhibits the power spectrum $P_{\omega}^{2}$, obtained by processing $P(t)$ recorded in a time window of $\Delta t=0.3 \mathrm{~s}$. duration in two seconds after switching on the driving force. A subharmonic at half frequency of the driving force can be seen apart from the main peak at the driving frequency and a turbulent cascade, which extents to $10 \mathrm{kHz}$. At frequencies

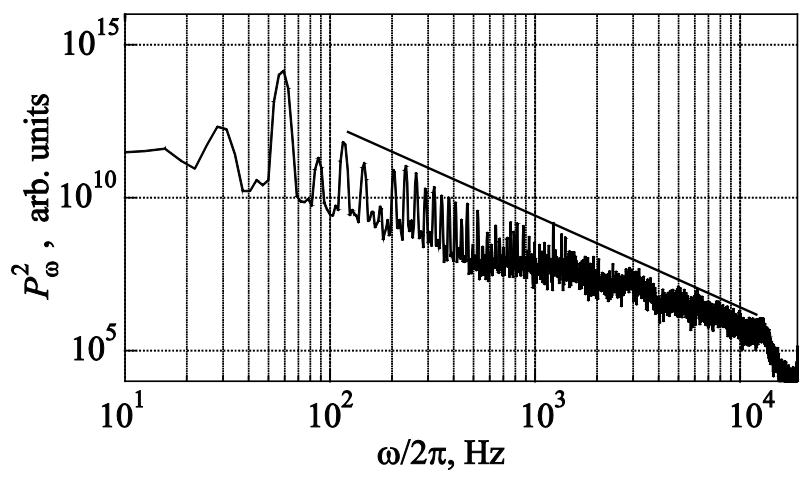

Fig. 1. Spectrum in two seconds after switching on the driving voltage at frequency of $58.6 \mathrm{~Hz}$. Straight line corresponds to the power-law dependence $\sim \omega^{-2.5}$. 
above $200 \mathrm{~Hz}$ the distribution $P_{\omega}^{2}$ displays the power-law dependence with the index $\beta \approx 2.5$, which is close to the theoretical prediction for the case of broadband pumping. The time of the subharmonic appearance in the distribution $P_{\omega}^{2}$ depends on the driving force amplitude and varies from a few seconds to several minutes after switching on the excitation.

A peak at the frequency of $\omega_{0} / 2$ on the turbulent cascade after 5 seconds of evolution from the moment of switching on the pumping is clearly visible in Fig. 2. The wave amplitude at the frequency $\omega_{0} / 2$ has not reached its maximum value yet, but it has already started affecting the turbulent distribution: peaks which are multiples of the half-harmonic frequency $\omega_{0} / 2$ start to emerge in the frequency range from 100 to $1000 \mathrm{~Hz}$. The height of the peaks in this frequency range can be described by the power function $\omega^{m}$ with an exponent close to -4.1 . Above $1 \mathrm{kHz}$ the height of the peaks in the spectrum $P_{\omega}^{2}$ decreases more slowly, in the frequency range from 1 to $10 \mathrm{kHz}$ it is described well by the power function with an exponent of -2.5 .

A local maximum on the distribution $P_{\omega}^{2}$ is clearly seen in the frequency domain from 10 to $20 \mathrm{kHz}$. Its amplitude is several times larger than the height of neighboring harmonics. At frequencies above $14 \mathrm{kHz}$ the turbulent cascade decays due to viscous losses. Note that during the evolution of the turbulent cascade the local maximum remains at the same position on the frequency scale.

Figure 3 presents the distribution $P_{\omega}^{2}$ formed on the surface in 30 seconds after switching on the excitation. Three peaks at frequencies $\omega_{0} / 2, \omega_{0}$ and $\omega_{0}+\omega_{0} / 2$, which dominate in the low-frequency domain play the role of pumping range, although the surface excitation by the electric force still goes on at a single frequency $\omega_{0}$. The turbulent distribution over the frequency range from $100 \mathrm{~Hz}$ to $20 \mathrm{kHz}$ can be described by the power law function with an exponent close to -2.8 . It is seen that at high frequencies the local extremum disappears, and the inertial interval

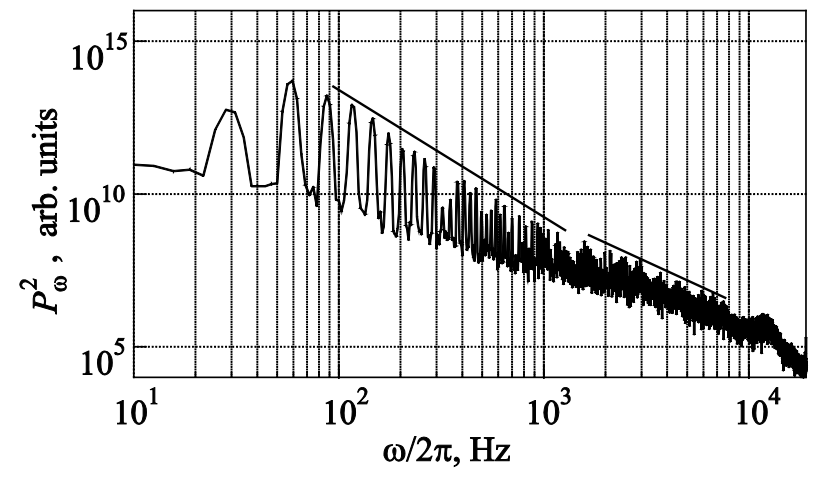

Fig. 2. Spectrum $P_{\omega}^{2}$ in five seconds after switching on the driving voltage. The solid lines corresponds to power-law functions $\sim \omega^{-4.1}$ and $\sim \omega^{-2.5}$.

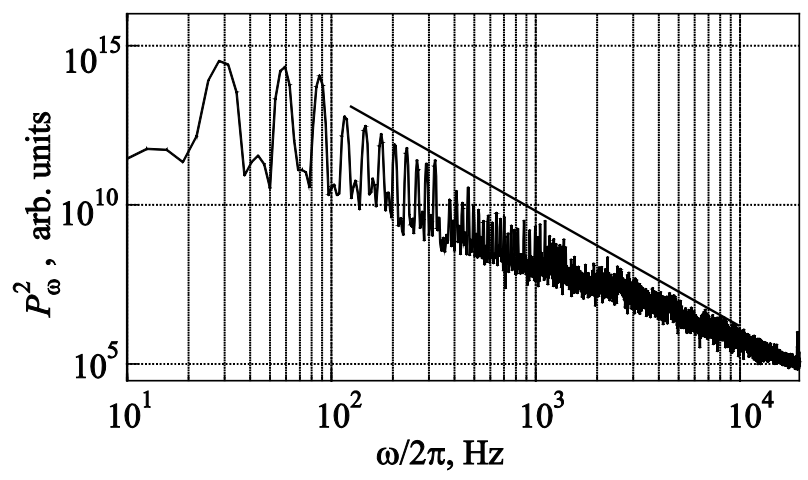

Fig. 3. Spectrum $P_{\omega}^{2}$ in thirty seconds after switching on the excitation. The solid line corresponds to power-law function $\sim \omega^{-2.8}$.

spreads to $20 \mathrm{kHz}$. The dissipative domain on the turbulent distribution is not seen.

The time evolution of the peaks at the driving force frequency $\omega_{0}$, at the frequency $\omega_{0} / 2$ and at frequency $\omega_{0}+\omega_{0} / 2$ is presented in Fig. 4 . The wave amplitude at the pumping frequency decreases by three times within the first 9 seconds after switching on the excitation. However, after 15 seconds of evolution its amplitude exceeds initial magnitude almost by 1.5 times.

The same time intervals can be distinguished for the subharmonic at $\omega_{0} / 2$. While its amplitude increases in both intervals, it grows much faster during the second time interval. The wave at frequency $\omega_{0}+\omega_{0} / 2$, appears simultaneously with the subharmonic and its amplitude gradually increases all $15 \mathrm{~s}$ from the beginning, and then remains almost constant. The vertical lines in Fig. 4 denote the time domain during which the local maximum on the turbulent distribution at high frequencies is observed. It can be emphasised that the local maximum exists during the first time interval when the harmonic amplitude at the driving frequency decreases, while the amplitude of the subharmonic increases.

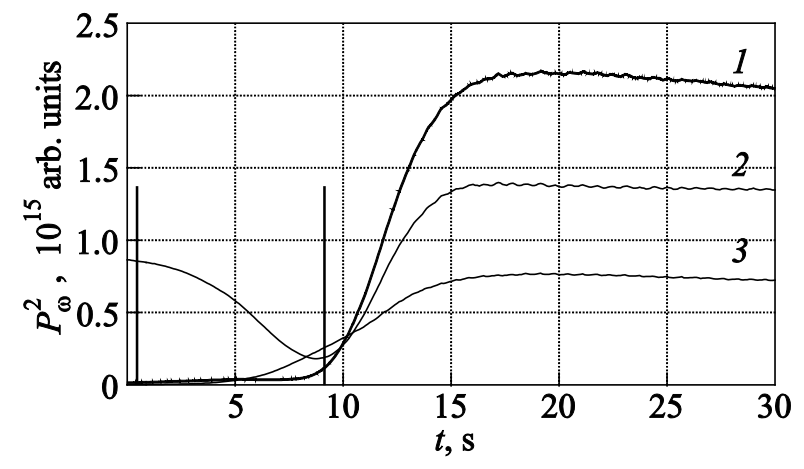

Fig. 4. Time dependence of amplitudes of the first three harmonics in the distribution of $P_{\omega}^{2}: \omega_{0} / 2$ (curve 1), $\omega_{0}$ (curve 2), $\omega_{0}+\omega_{0} / 2$, (curve 3 ), $\omega_{0} / 2 \pi=58.6 \mathrm{~Hz}$. Vertical lines mark the time interval when the local maximum on the turbulent cascade can be identified. 


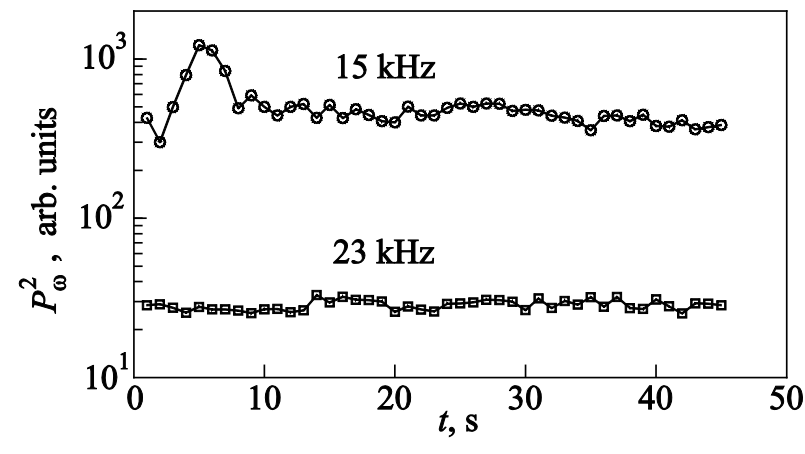

Fig. 5. Time dependence of amplitudes of harmonics at frequencies 15 and $23 \mathrm{kHz}$.

Figure 5 shows two cases of time dependence of the peak amplitude at frequencies 15 and $23 \mathrm{kHz}$ after switching on pumping. The peak at $15 \mathrm{kHz}$ is in the middle of the dissipation domain and the peak at the frequency of $23 \mathrm{kHz}$ - beyond the dissipation area. It is seen that the peak amplitude at the frequency of $15 \mathrm{kHz}$ after switching on the excitation increases almost by three times for the first 5 seconds, then decreases and reaches a steady state level at the 10th second. The amplitude of the peak at the frequency of $23 \mathrm{kHz}$ is practically independent of time, that is, the amplitude is not sensitive to switching on the excitation force.

\section{Discussion}

All spectra of capillary turbulence presented in this paper were obtained under excitation of the surface oscillations by an external harmonic force. Although the spectrum of the eigenfrequencies of the surface oscillations in a finite basin is of discrete nature, it can be considered quasicontinuous above several kilohertz due to viscous broadening of the eigenmodes. When the surface oscillation is excited by a monochromatic pumping, a turbulent cascade is formed which consists of a multiple harmonic of a pumping frequency and extends for more than two decades. The appearance of the first subharmonic mode at half the driving frequency leads to a reconstruction of the cascade and is accompanied by the formation of a local maximum in the power spectrum near the high frequency boundary of the inertial range. However, the maximum manifests itself for a period of several seconds, and disappears completely before the turbulent cascade reaches its new steady state distribution. The time of local maximum existence coincides with the time of the decrease of the main harmonic amplitude at the driving frequency and growth of the subharmonic and combination harmonics (Fig. 4).

The observed phenomenon cannot be explained as related to the formation of a bottleneck preventing the energy transfer towards high frequencies due to the detuning effect in discrete systems as in the case of liquid helium experiment [7], because the eigenmode spectrum of the surface oscillations is quasi-continuous above $4 \mathrm{kHz}$. The influence of discreetness on the turbulent distribution in the system of waves on the liquid hydrogen surface was studied before in $[10,11]$.

The formation of the local maximum on the turbulent cascade near the high frequency boundary may be attributed to the bottleneck effect caused by the viscous damping in a high frequency domain. As was shown in [8] the finiteness of the dissipation scale leads to an increase of the wave amplitudes in the inertial range. The energy accumulation near the high frequency edge of the inertial range is caused by a reduction of the energy flux due to low occupation numbers (waves amplitudes) in the dissipation domain. Though the original bottleneck effect [9] was described for steady state spectra of turbulence, the same rationale can be extended to our dynamic case. Note that the local maximum is observed during an intensive energy loss of the main harmonic (Fig. 5), the main harmonic releases about $90 \%$ of its initial energy during the first 5 seconds. Under a permanent pumping by the external force this energy loss cannot be related to the viscous dissipation, but to the nonlinear energy transfer to the growing subharmonic and multiple harmonics. The viscous damping (Eq. 4) of waves within the inertial range is not sufficient to dissipate the released energy, and the energy is transferred to higher frequencies by the nonlinear wave interaction towards the dissipation domain, where the mechanism of the bottleneck begins to act. The delay between the growth of the subharmonic at half of the driving frequency and the appearance of the local maximum at high frequencies enables the estimation of the velocity of local disturbance propagation over the turbulent cascade as $d \omega / d t \sim \omega / t \sim 10^{4} \mathrm{~Hz} / \mathrm{s}$.

\section{Conclusion}

We experimentally observed the accumulation of energy near the high frequency boundary of the inertial range on the spectrum of turbulence because of reduction of energy flux in dissipation domain - the bottleneck effect.

Authors are grateful to G.V. Kolmakov and L.P. Mezhov-Deglin for useful discussions. This work was supported in part by the program project of the Presidium of the Russian Academy of Sciences «Modern problems of low temperature physics».

1. V.E. Zakharov, V.S. L’vov, and G. Falkovich, Kolmogorov Spectra of Turbulence I, Springer-Verlag (1992).

2. G. Kolmakov, M.Yu.Brazhnikov, A.A. Levchenko, L.V. Abdurakhimov, P.V.E. McClintock, and L.P. Mezhov-Deglin, Capillary Turbulence on the Surfaces of Quantum Fluids, in: Progress in Low Temperature Physics, Quantum Turbulence 16 (2009).

3. E.A. Kartashova, Physica D 46, 43 (1990).

4. G. Falkovich and A. Shafarenko, Sov. Phys. JETP 67, 1393 (1988). 
5. V. Zakharov and N.Filonenko, J. App. Mech. Tech. Phys. 8, 62 (1967).

6. A. Pushkarev and V. Zakharov, Physica D 135, 98 (2000).

7. L.V. Abdurakhimov, M.Yu. Brazhnikov, I.A. Remizov, and A.A. Levchenko, Pisma v ZhETP 91, 291 (2010).

8. I. Ryzhenkova and G. Falkovich, Sov. Phys. JETP 71, 1085 (1990).
9. M. Brazhnikov, A. Levchenko, and L. Mezhov-Deglin, Instr. Exp. Tech. 45, 758 (2002).

10. M.Y. Brazhnikov, A.A. Levchenko, L.P. Mezhov-Deglin, and I.A. Remizov, Fiz. Nizk. Temp. 41, 615 (2015) [Low Temp. Phys. 41, 484 (2015)].

11. L.V. Abdurahimov, M.Yu. Brazhnikov, A.A. Levchenko, I.A. Remizov, Fiz. Nizk. Temp. 41, 163 (2015) [Low Temp. Phys. 41, 215 (2015)]. 